\title{
Conocimientos y prácticas sobre salud bucal en profesores de dos escuelas: Quito y San Cristóbal (Galápagos), Ecuador
}

\author{
Knowledge and practices about oral health among teachers in two schools: Quito and San \\ Cristobal island (Galapagos), Ecuador
}

Ana Armas Vega', Andrés Viteri-García², Luis C. Castillo³, Andrés A. Agudelo-Suárez ${ }^{4}$

\begin{abstract}
RESUMEN
Objetivo: Analizar los conocimientos, actitudes y prácticas que sobre salud oral tienen los profesores de educación primaria de centros escolares de Quito y de la Isla San Cristóbal (Galápagos), Ecuador. métodos: La investigación se inició con la validación de la encuesta autodiligenciada sobre conocimientos, actitudes y prácticas sobre salud bucal en docentes (CAPS docentes). Posteriormente este instrumento fue entregado a 61 docentes de los dos centros escolares seleccionados para el estudio. Se obtuvo la estadística descriptiva de estos datos, que incluyó las frecuencias absolutas y relativas de las respuestas obtenidas. Resultados: La media de la puntuación obtenida en evaluación total de los 61 docentes fue de $11,97 \pm 2,37$ puntos. Cuando se analizaron los datos por centros escolares, la escuela de Quito obtuvo una media de 12,06 $\pm 2,29$, mientras que la de Galápagos obtuvo una media de 11,29 $\pm 3,04$ puntos. Conclusiones: El conocimiento en salud bucal de los docentes de los dos centros educativos fue aceptable, no se observó una diferencia en la puntuación de la evaluación total debido a la procedencia de los mismos.
\end{abstract}

Palabras clave: Conocimientos; Actitudes y Práctica en Salud; Salud Bucal. (Fuente: DeCS BIREME)

\begin{abstract}
Objective: To analyze the knowledge, attitudes and practices that oral health teachers have in primary schools in Quito and San Cristobal Island (Galapagos), Ecuador. Methods: The research began with the validation of the self-surveyed survey on knowledge, attitudes and practices on oral health in teachers (CAPS teachers). Subsequently, this instrument delivered to 61 teachers from the two schools selected for the study. The descriptive statistics of these data were obtained, including the absolute and relative frequencies of the responses obtained. Results: The average score obtained in the total evaluation of the 61 teachers was $11.97 \pm 2.37$ points. When the data were analyzed by schools, the Quito school obtained an average of $12.06 \pm 2.29$, while the Galapagos school obtained an average of $11.29 \pm 3.04$ points. Conclusions: The knowledge in oral health of the teachers of the two educational centers was acceptable; there was no difference in the score of the total evaluation due to their origin.
\end{abstract}

Keywords: Knowledge; Attitudes and Practice in Health; Oral Health. (Source: MeSH NLM)

${ }^{1}$ Centro de Investigación en Salud Oral (CISO). Facultad de Ciencias de la Salud "Eugenio

Espejo". Universidad UTE. Quito-Ecuador

${ }^{2}$ Centro de Investigación en Salud Pública y Epidemiología Clínica (CISPEC). Facultad de

Ciencias de la Salud "Eugenio Espejo". Universidad UTE. Quito-Ecuador.

${ }^{3}$ Universidad Central del Ecuador. Quito-Ecuador

${ }^{4}$ Facultad de Odontología. Universidad de Antioquia. Medellín-Colombia

Este es un artículo Open Access distribuido bajo la licencia Creative Commons Atribución-No ComercialCompartir Igual 4.0

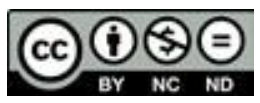

Correspondencia

Andrés Viteri-García

Centro de Investigación en Salud Pública y Epidemiología Clínica (CISPEC). Facultad de Ciencias de la Salud "Eugenio Espejo". Universidad UTE.

Av. Mariana de Jesús S/N y Av. Mariscal Sucre, Quito-Ecuador. Teléfono: +593 992836303

Correo electrónico: andres.viteri@ute.edu.ec

Citar como: Armas A. Viteri A. Castillo L. Agudelo A. Conocimientos y prácticas sobre salud bucal en profesores de dos escuelas: Quito y San Cristóbal (Galápagos), Ecuador. KIRU. 2020; ene-mar 17(1): $16 \quad$ - 22. https://doi.org/10.24265/kiru.2020. v17n1.03 


\section{INTRODUCCIÓN}

La caries dental es un problema de salud pública que puede entenderse como un proceso infeccioso, paulatino, que desencadena la destrucción de los tejidos duros ${ }^{(1,2)}$. En este proceso la influencia de las condiciones sociales y ambientales en las que el individuo se desarrolla son de suma importancia ${ }^{(3,4)}$, por lo que se han desarrollado varias estrategias a nivel local, regional y nacional para su control, desde una perspectiva de salud pública. ${ }^{5}$

Reportes, como el presentado por la OMS en 2012, refieren que a nivel mundial del $60 \%$ al $90 \%$ de los niños en edad escolar tienen caries dental|(5), a menudo acompañada de dolor y sensación de molestia(6). En Ecuador, el estudio epidemiológico realizado en el 2009 refiere una prevalencia de caries dental no tratada de un $75,6 \%$ de la población escolar entre 6 a 15 años de edad que se incrementa proporcionalmente con la $\operatorname{edad}^{(7)}$. Esta situación evidencia la alta vulnerabilidad de esta población a la enfermedad. ${ }^{8}$

Pese al impulso de acciones dirigidas a la prevención de la caries dental proporcionado por las diferentes entidades de salud a nivel mundial, aún se percibe cierto desinterés y falta de conocimiento, especialmente en los niveles socioculturales bajos ${ }^{(9)}$. Esta situación lleva a considerar a la educación para la salud bucal como el pilar fundamental para la sostenibilidad de programas y estrategias ${ }^{(10)}$, ya que al modificar actitudes y motivar en los individuos la adquisición de hábitos bucales correctos, se promueve el control de diferentes enfermedades bucales $^{(11)}$.

La falta de conocimientos sobre hábitos adecuados de higiene bucal y de alimentación constituye uno de los principales factores que explican la ocurrencia de esta enfermedad ${ }^{(12)}$, de ahí que, medidas de educación en salud bucal direccionadas a padres y maestros como responsables de esta población resultan indispensables ${ }^{(13)}$.

El personal odontológico se muestra como un agente importante para la creación de hábitos y conductas saludables ${ }^{(14)}$, mediante técnicas educativas y afectivoparticipativas ${ }^{(15)}$. Diferentes estudios resaltan el éxito conseguido al contar con escolares motivados y con conocimiento sobre normas de higiene y salud para su cumplimiento diario ${ }^{(16,17)}$ y que al ser instauradas logran responsabilizar al sujeto de su propia salud logrando que adopte estilos de vida mucho más saludables ${ }^{(4)}$ Un punto importante para la realización de programas y estrategias en salud bucal, es conocer las propias necesidades e intereses de los individuos con relación a su contexto social ${ }^{(18)}$, esto con el fin de identificar debilidades y fortalezas.

Los profesores de las instituciones educativas pueden ser importantes agentes de cambio, aliados en las estrategias y programas de salud bucal, como lo demostrado en diferentes estudios ejecutados en otros contextos locales ${ }^{(16,1)}$; no obstante, las evaluaciones, tomando como base conocimientos, actitudes y prácticas en el territorio ecuatoriano, son escasas. El objetivo de este estudio fue analizar los conocimientos, actitudes y prácticas sobre salud bucal de profesores de educación primaria de centros escolares de Quito y de la Isla San Cristóbal (Galápagos), Ecuador.

\section{MÉTODOS}

Se realizó un estudio observacional, transversal y descriptivo, en una muestra por conveniencia de 61 docentes de educación primaria de centros escolares de la ciudad de Quito y de San Cristóbal en las Islas Galápagos, Ecuador. El período de reclutamiento y recolección de las encuestas se realizó en dos meses del año 2017, se contó con el consentimiento informado de las autoridades y docentes de las instituciones educativas.

Se aplicó un cuestionario autodiligenciado sobre conocimientos, prácticas y actitudes en salud bucal; cuestionario basado en 20 preguntas, cada una con 4 respuestas de opción múltiple, de las cuales solo una es correcta. La puntuación de esta herramienta tiene un rango de 0 a 20 puntos. Cabe aclarar que este instrumento (Anexo 1) fue adaptado del cuestionario "Conocimientos de los padres sobre la salud bucal de niños re escolares: desarrollo y validación de un instrumento" de Cupé-Araujo y García-Rupaya $(2015)^{(19)}$, desarrollado y validado en español y aplicado a padres de familia.

El proceso de validación de la encuesta consistió en la entrega del cuestionario a 10 docentes de centros escolares, que no participaron posteriormente en el estudio, para comprobar el nivel de comprensión de las preguntas, los resultados de este proceso fueron muy aceptables, puesto que todos los participantes contestaron todas las preguntas y explicaron que habían entendido claramente las mismas. Inmediatamente después, se realizó el pilotaje del cuestionario con 30 docentes a los cuales se les asignó un código aleatorio para, después de 15 días, volver a aplicarlo; se realizó un análisis de concordancia test-retest y se obtuvo un alfa de Cronbach de 0,76 considerado como fiable. 
Posterior a esta validación, se aplicó el cuestionario a los 61 docentes ( $n=54$ en Quito, $n=7$ en Galápagos)

Los datos de esta evaluación fueron recolectados en fichas identificadas con códigos para mantener el anonimato de los participantes. Los datos fueron tabulados en Excel, para ser procesados a partir de la estadística descriptiva y realizar su análisis, mediante frecuencias absolutas y relativas.

\section{RESULTADOS}

Se entrevistaron 61 docentes, la mediana de edad fue de 42 años (IQR: 53-34) y en promedio el resultado total de la evaluación fue de 11,97 $\pm 2,37$ puntos. La tabla 1 resume los valores de acuerdo a la ciudad de origen de los docentes.

tabla 1. Resultados generales por ciudad.

\begin{tabular}{ccc}
\hline & $\begin{array}{c}\text { Quito } \\
(\mathbf{n}=54)\end{array}$ & $\begin{array}{c}\text { San Cristóbal } \\
(\mathbf{n}=7)\end{array}$ \\
\hline $\begin{array}{c}\text { Edad (años): } \\
\widetilde{\boldsymbol{x}}(\mathrm{IQR})\end{array}$ & $40(49-34)$ & $55(58-34)$ \\
\hline$\%$ Docentes evaluados & $88,5 \%$ & $11,5 \%$ \\
\hline $\begin{array}{c}\text { Resultado total } \\
\text { total } \overline{\boldsymbol{x}} \pm \mathrm{SD}\end{array}$ & $12,06 \pm 2,29$ & $11,29 \pm 3,04$ \\
\hline
\end{tabular}

IQR = Rango intercuartílico, $\widetilde{x}=$ media, $\mathrm{SD}=$ Desviación estándar

Las preguntas en las que con mayor frecuencia los docentes respondieron de manera incorrecta fueron las relacionadas con: el conocimiento de qué es la caries dental $(n=59 ; 96,7 \%)$, placa bacteriana $(n=45$; $73,8 \%$ ), la edad y presencia de dientes de leche $(\mathrm{n}=41 ; 67,2 \%)$, la edad de la primera visita al odontólogo ( $n=34 ; 55,7 \%)$, el número de veces al día en que el niño debe cepillarse los dientes $(n=54 ; 88,5$ $\%)$ y la que evalúa desde que edad se puede usar pasta dental con flúor $(n=41 ; 67,2 \%)$.
Las preguntas relacionadas con la importancia de los dientes temporales, la que evalúa la necesidad de visitar al dentista, los alimentos adecuados para tener dientes sanos y la que aborda la alimentación recomendada para llevar en la lonchera, son las preguntas a las cuales más del $90 \%(n>55)$ de los docentes contestaron correctamente (Figura 1).

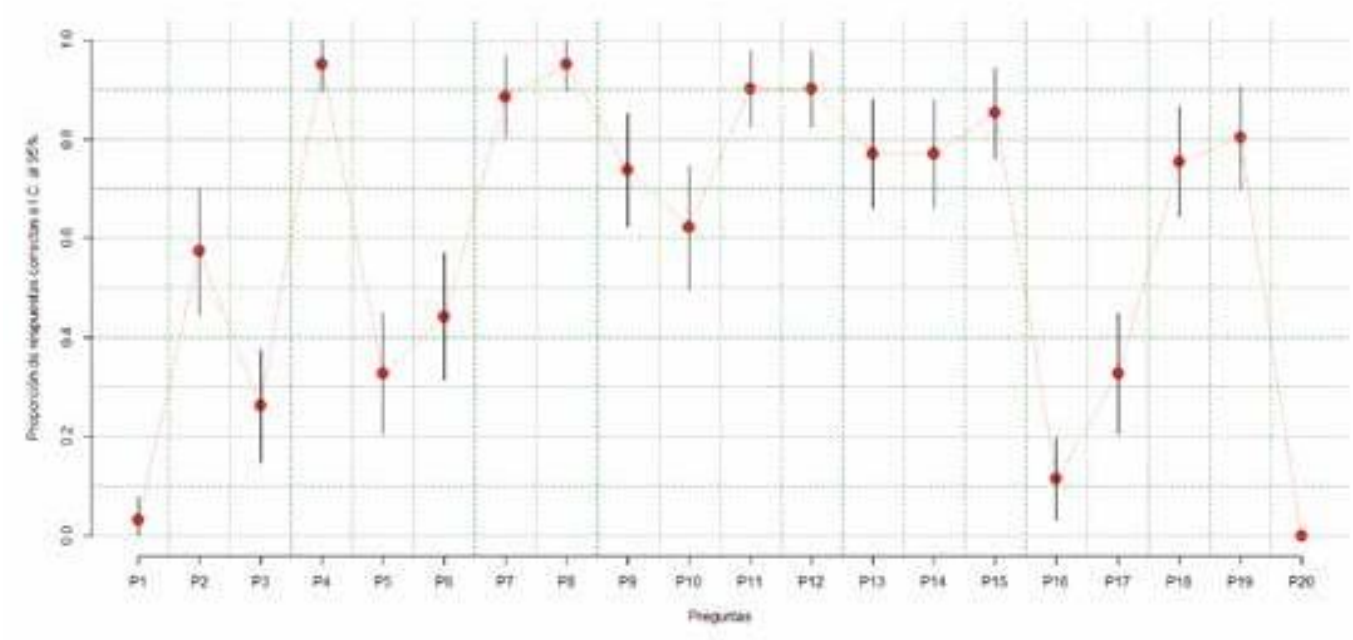

Figura 1. Proporción de docentes que contestaron correctamente las preguntas evaluadas en los centros escolares encuestados $(\%$, IC). 
Al contabilizar el número de preguntas que fueron contestadas correctamente, se encontró que en promedio los docentes, independientemente de la procedencia, aciertan alrededor del $60 \%$ de las preguntas.

\section{DISCUSIÓN}

Los resultados evidenciaron un conocimiento moderado sobre salud bucal, sin distinción entre las dos ciudades participantes, situación que se explicaría, en parte, debido a la ausencia temas relacionados con la higiene oral y salud bucodental en el currículo de los programas académicos en las instituciones formadoras de los recursos humanos para la educación; asociado a esto, posiblemente a la poca o nula importancia a las estrategias de educación de salud oral que de forma personal se cultiven $^{(17)}$.

Las diferentes estrategias de control de la lesión cariosa enfatizan la importancia que los procesos educativos tienen como estrategia de control de la enfermedad. ${ }^{4}$ Los profesores de centros escolares primarios son responsables indirectos de una gran cantidad de niños en edades de alta susceptibilidad de recepción y adquisición de hábitos, el docente se convierte en la persona que mayor influencia tiene sobre el niño después de sus padres ${ }^{(20)}$. Es por ello que resulta esencial identificar el nivel de conocimientos que los docentes tienen sobre la salud bucal y evidenciar cómo a través de la intervención con programas de educación para la salud se puede modificar sus acciones, lo que conlleva a un acercamiento con el grupo de profesionales de la odontología direccionados hacia los niños de los centros escolares.

La efectividad de los diferentes programas educativos sobre la salud bucal en los que se consideran los cambios de actitud y hábitos bucales, cuentan con una gran aprobación de por parte de los niños intervenidos, como lo muestran las políticas a nivel nacional de salud dental bucal a 10 años de seguimiento en Brasil. ${ }^{21}$ Liontou y colaboradores mostraron que a pesar de la falta de conocimientos sobre higiene oral de un grupo de maestros de preescolar, estos reconocieron la importancia de la misma y estaban dispuestos a participar en los programas educativos ${ }^{(20)}$.

Al ser la caries dental una enfermedad de desarrollo lento y susceptible de revertir(10); la incorporación de estrategias de higiene bucal se muestra como una gran herramienta ${ }^{(18)}$ para limitar el daño con el propósito de detener la enfermedad y controlar las secuelas físicas, emocionales, familiares e incluso económicas ${ }^{(20,22)}$. Por lo que se debe incluir en ellas a los responsables directos de la ejecución diaria de actividades de remoción de la placa y reversión de la lesión en sus etapas incipientes ${ }^{(11)}$, por lo que resulta básico considerar al profesorado de los centros escolares como aliados en este trabajo ${ }^{(15)}$.

Una limitación en el presente estudio es la población reducida, tanto en docentes como en centros escolares. No obstante, permite tener una primera aproximación de la realidad que se aprecia en el día a día de los centros escolares con respecto a los conocimientos y prácticas relacionas con salud bucal, por lo que es necesario realizar estudios con mayor rigurosidad metodológica, para poder obtener datos que permitan emitir sugerencias de reforma en los currículos de las instituciones formadoras de docentes de educación básica y elemental.

\section{CONCLUSIONES}

Se evidenció que el conocimiento en salud bucal de los docentes de las dos ciudades fue aceptable. Se requiere desarrollar estrategias que permitan que los conocimientos de los docentes aumenten; y, sobre todo, que sus prácticas y actitudes respecto a la salud bucodental se optimicen.

Contribuciones de autoría: DSM, JPRV, AA, EV, VBB y MF diseñaron el estudio, recopilaron y analizaron los datos, redactaron y aprobaron el manuscrito.

Fuente de financiamiento: Autofinanciado.

Conflicto de intereses: Los autores declararon no tener conflictos de interés.

\section{REFERENCIAS}

1. Singhal RK, Rai B. Remineralization Potential of Three Tooth Pastes on Enamel Caries. Open Access Maced J Med Sci. 2017 Jul 29;5(5):664-666. doi: 10.3889/ oamjms.2017.090. eCollection 2017 Aug 15.

2. Philip N, Suneja B, Walsh LJ. Ecological Approaches to Dental Caries Prevention: Paradigm Shift or Shibboleth? Caries Res. 2018 Jan 11;52(1-2):153-165. doi: 10.1159/000484985. [Epub ahead of print]

3. Collins CC, Villa-Torres L, Sams LD, Zeldin LP, Divaris K. Framing Young Childrens Oral Health: A Participatory Action Research Project. PLoS One. 2016 Aug:22;11(8):e0161728. doi: 10.1371/journal. pone.0161728.

4. Pinto GDS, Azevedo MS, Goettems ML, Correa MB, Pinheiro RT, Demarco FF. Are Maternal Factors Predictors for Early Childhood Caries? Results from a Cohort in Southern Brazil. Braz Dent J. 2017 MayJun;28(3):391-397. doi: 10.1590/0103-6440201601047. 
5. Organización Mundial de la Salud. (2012). Salud Bucodental, nota informativa No. 318. [Fact sheet]. Disponible en: http://www.who.int/mediacentre/ factsheets/fs318/es/

6. Musinguzi N, Kemoli A, Okullo I. Prevalence and Treatment Needs for Early Childhood Caries Among 35-Year-Old Children From a Rural Community in Uganda. Front Public Health. 2019 Sep 18;7:259. doi: 10.3389/fpubh.2019.00259.

7. Raza X, Alvear A, Andrade R, Ayala E, Chilliquinga M, Luque I, et al. Estudio Epidemiológico Nacional de Salud Bucal en Escolares Menores de 15 años del Ecuador. 2010. MSP/OPS.

8. Aguiar VR, Pattussi MP, Celeste RK. The role of municipal public policies in oral health socioeconomicinequalities in Brazil: A multilevel study. Community Dent Oral Epidemiol. 2017 Dec 7. doi:10.1111/cdoe.12356. [Epub ahead of print]

9. Kassebaum NJ, Smith AGC, Bernabé E, Fleming TD, Reynolds AE, Vos T, Murray CJL, Marcenes W. GBD 2015 Oral Health Collaborators. Global, Regional, and National Prevalence, Incidence, and Disability-Adjusted Life Years for Oral Conditions for 195 Countries, 19902015: A Systematic Analysis for the Global Burden of Diseases, Injuries, and Risk Factors. J Dent Res. 2017 Apr;96(4):380-387. doi: 10.1177/0022034517693566.

10. Dos Santos AP, Nadanovsky $P$, de Oliveira BH. A systematic review and meta-analysis of the effects of fluoridetoothpastes on the prevention of dental caries in the primary dentition of preschool children. Evid Based Dent. 2014; 1: 5. Evid Based Dent. 2014 Sep;15(3):67. doi: $10.1038 /$ sj.ebd.6401035.

11. Mertz E, Wides C, White J. Clinician attitudes, skills, motivations and experience following the implementation of clinical decision support tools in a large dentalpractice. J Evid Based Dent Pract. 2017 Mar;17(1):1-12. doi: 10.1016/j.jebdp.2016.10.001. Epub 2016 Oct 20.

12. Bhayade SS, Mittal R, Chandak S, Bhondey A. Assessment of social, demographic determinants and oral hygiene practices in relation to dental caries among the children attending Anganwadis of Hingna, Nagpur. J Indian Soc Pedod Prev Dent. 2016 Apr-Jun;34(2):124-7. doi: 10.4103/0970-4388.180415.

13. Baldani $\mathrm{MH}$, Rocha JS, Fadel CB, Nascimento AC, Antunes JLF, Moysés SJ. Assessing the role of appropriate primary health care on the use of dental services by Brazilian low-income preschool children. Cad Saude Publica. 2017 Nov 21;33(11):e00158116. doi: 10.1590/0102-311X00158116
14. Phantumvanit $P$, Makino $Y$, Ogawa $H$, Rugg-Gunn $A$, Moynihan P, Petersen PE, Evans W, Feldens CA, Lo E, Khoshnevisan MH, Baez R, Varenne B, Vichayanrat T, Songpaisan Y, Woodward M, Nakornchai S, Ungchusak C. WHO Global Consultation on Public Health Intervention against Early Childhood Caries. Community Dent Oral Epidemiol. 2018 Jan 30. doi: 10.1111/cdoe.12362. [Epub ahead of print]

15. Cisneros-Domínguez G, Hernández-Borges Y. La educación para la salud bucal en edades tempranas de la vida. MEDISAN. 2011 Oct:15(10):1445-1458.

16. Das UM, Vadakkekuttical RJ, Kanakkath $H$, Shankunni SP. Dental health awareness, attitude, and dental health-care seeking practices as risk indicators for the prevalence of periodontal disease among 15-17-yearold school children in Kozhikode district, Kerala, India. J Indian Soc Periodontol. 2017 Mar-Apr;21(2):144-151. doi: 10.4103/jisp.jisp_160_17.

17. Kaewkamnerdpong I, Krisdapong S. The Associations of School Oral Health-Related Environments with Oral Health Behaviours and Dental Caries in Children. Caries Res. 2018 Jan 23;52(1-2):166-175. doi: 10.1159/000485747. [Epub ahead of print]

18. Patel PM, Hugar SM, Halikerimath S, Badakar CM, Gokhale NS, Thakkar PJ, Kohli D, Shah S. Comparison of the Effect of Fluoride Varnish, Chlorhexidine Varnish and Casein Phosphopeptide- Amorphous Calcium Phosphate (CPP-ACP) Varnish on Salivary Streptococcus mutans Level: A Six Month Clinical Study. J Clin Diagn Res. 2017 Aug;11(8):ZC53-ZC59. doi: 10.7860/JCDR/2017/26541.10409. Epub 2017 Aug 1.

19. Cupé-Araujo AC, Garcia-Rupaya CR. Conocimientos de los padres sobre la salud bucal de niños pre escolares: desarrollo y validación de un instrumento. Rev Estomalo Herediana 2015, 25(2):112-121.

20. Liontou V, Agouropoulos A, Gizani S, Papagiannoulis L. Knowledge of preschool teachers in the prefecture of Attica of early childhood oral health. Association with their demographic and personal characteristics. Eur Arch Paediatr Dent. 2016 Dec;17(6):467-474. Epub 2016 Nov 17.

21. Pucca GA, Gabriel ME, de Araujo ME, de Almeida FCS Ten Years of a national oral health policy in Brasil. $J$ Dental Research. 2015. 94(10):1333-7 doi: 10.1177/0022034515599979. Epub 2015 Aug 27.

22. Silveira ML, Dye BA, lafolla TJ, Adesanya MR, Boroumand S, Youngblood ME, Salazar CR, Finlayson TL, Khambaty T, Beaver SM, Garcia Al. Cultural factors and oral health-related quality of life among dentate adults: Hispanic community health study/ study of Latinos. Ethn Health. 2018 Jan 18:1-16. doi: 10.1080/13557858.2018.1427219. 


\section{anexo 1. Encuesta utilizada en los docentes de dos centros educativos}

1. ¿Qué es la caries dental?

a) Es una enfermedad que aparece en los niños desnutridos.

b) No es una enfermedad

c) Es una enfermedad causada por falta de higiene bucal y consumo de azúcares.

d) Es una enfermedad causada por falta de higiene bucal y consumo de frituras.

2. Los microorganismos que causan la caries dental puede transmitirse por:
a) Compartir utensilios
b) Compartir cepillos dentales
c) Besos en la boca
d) Todas las anteriores

3. ¿Qué es la placa bacteriana dental?
a) Es una capa dura que se forma en la superficie de los dientes
b) Es una capa blanda que se forma en la superficie de los dientes
c) Es el sarro que se forma en los dientes
d) Es una masa que solo se encuentra en los dientes de los adultos

4. ¿Son importantes los dientes de leche?
a) Sí, porque guardan espacio para los dientes permanentes
b) No, porque no cumplen ninguna función
c) No, porque al final se van a caer
d) No, porque no son los dientes permanentes

5. ¿A qué edad los niños tienen todos los dientes de leche?
a) 2 años
b) 4 años
c) 6 años
d) 8 años

6. La primera visita al odontólogo, se recomienda a partir de:
a) A partir de los 2 años
b) cuando aparece el primer diente de leche
c) Cuando tenemos dientes de adulto
d) Solo cuando existe dolor

7. ¿Qué beneficios conoce del flúor?
a) Fortalece los dientes y previene la caries
a) Cura los dientes para prevenir las extracciones
a) El flúor tiene una acción blanqueadora en los niños
a) Fortalece al niño para que crezca sano y fuerte

8. Es necesario visitar al dentista cuando se tiene dientes sanos:
a) Sí, para un examen clínico de rutina
a) Solo si tiene dientes chuecos
a) No, ya que como no tiene nada no es necesario
a) Solo voy si yo o alguien de mi familia tiene molestias en la boca

9. Si su niño pierde un diente de leche, antes del tiempo porque está con caries ¿cree Ud. quepuede afectar la posición de los dientes?
a) Sí
a) Depende, solo si pierde las muelas de leche
a) No
a) Los dientes de leche no son importantes

10. ¿Qué medidas preventivas conoce usted para combatir la caries dental?
a) El flúor
a) Una correcta higiene bucal
a) Evitar consumo excesivo de azúcares
a) Todas las anteriores

11. ¿Qué alimentos cree usted que son mejores para tener dientes más sanos?
a) Frutas y vegetales
b) Gaseosas y frutas
c) Jugos y galletas
d) Todas las anteriores

12. ¿Qué se recomienda que lleve un niño en la lonchera?
a) Queso, quinua, frutas, huevo, pan, carne
b) Galletas dulces, chocolates, tortas, refrescos de caja
c) Jugos de cartón, leche chocolatada, chitos
d) Todas las anteriores 
13. Con respecto al consumo de azucares, marque lo correcto

a) El niño nunca debe consumir azúcar

b) El niño puede consumir azúcar en varios momentos durante el día

c) El niño puede consumir azúcar en horas determinadas y luego cepillarse los dientes

d) Todas las anteriores

14. ¿Hasta qué edad se recomienda dar al niño lactancia materna?
a) Hasta los 15 días de nacido
b) Hasta los 3 meses de edad
c) Hasta los 6 meses de edad
d) Hasta el primer mes de nacido

15. Dejar que el niño tenga el biberón con líquidos azucarados durante toda la noche ¿qué causaría?
a) Va a estar más fuerte al despertar
b) Estará más fuerte y sano
c) Estará más expuesto a tener caries
d) No pasa nada

16. ¿Cuántas veces al día el niño debe cepillarse los dientes?
a) 1 vez
b) De 2 a 3 veces
c) De 5 a más veces
d) Los niños no deben cepillarse los dientes

17. ¿Desde qué edad se puede usar pasta dental con flúor?
a) A partir de los 2 años
a) A partir de 5 años
a) A partir de la adolescencia
a) En niños menores de 2 años

18. ¿El cepillado se recomienda realizarlo después de cada comida?
a) $\mathrm{No}$
a) Solo antes de dormir
a) Solo con usar un enjuagatorio basta
a) Sí

19. ¿Cada qué tiempo se debe cambiar el cepillo dental?
a) Cada 3 meses
a) Cada 8 meses
a) Alaño
a) Nunca

20. En cuanto al cepillado dental

a) El cepillado dental puede ser realizado solo por el mismo niño

a) Los padres deben supervisar el cepillado dental de sus niños a esta edad

a) El cepillado dental debe ser realizado en niños mayores de 3 años.

a) En el niño el cepillado dental debe realizarse sin pasta dental
Diego Xavier Schmiedl Manzano diegorb_@live.com

Juan Pablo Rodríguez Villarreal pablorodri-@hotmail.es

Ana del Carmen Armas

ana_del_ec@yahoo.es

Estefanía Viteri

saviteris93@hotmail.com

Valeria Elizabeth Banderas Benitez

Valeria.banderas.benitez@gmail.com

Maria Elena Flores

me.flores@udlanet.ec
$0000-0002-5554-9426$

0000-0001-8191-9593

$0000-0003-3800-8166$

0000- 0002-2929-1759

(D) $0000-0002-6467-4958$

0000-0002-8502-4102 